\title{
Téoros
}

Revue de recherche en tourisme

\section{Les voyages des Américains aux États-Unis}

\section{Elizabeth Stewart}

Volume 9, numéro 2, juillet 1990

Le marché américain

URI : https://id.erudit.org/iderudit/1080025ar

DOI : https://doi.org/10.7202/1080025ar

Aller au sommaire du numéro

Éditeur(s)

Université du Québec à Montréal

ISSN

0712-8657 (imprimé)

1923-2705 (numérique)

Découvrir la revue

Citer cet article

Stewart, E. (1990). Les voyages des Américains aux États-Unis. Téoros, 9(2),

9-13. https://doi.org/10.7202/1080025ar d'utilisation que vous pouvez consulter en ligne.

https://apropos.erudit.org/fr/usagers/politique-dutilisation/ 


\section{Les voyages des Américains aux Etats-Unis}

L'industrie des voyages et du tourisme est l'une des principales industries aux ÉtatsUnis. Environ 1,3 milliard de voyagespersonnes ont été effectués en 1989, générant 333,6 milliards de dollars en recettes au niveau des entreprises dont 1'activité principale est liée aux voyages. En 1980 , seulement 1,04 milliard de voyages-personnes ont été effectues, pour des recettes de 171,8 milliards de dollars. Cette croissance a profite a toutes les principales composantes de l'industrie. Le secteur hôtelier a grossi et s'est développé, les compagnies aériennes se sont multipliées et ont ensuite fusionné, et les stimulants visant à inciter les consommateurs à la fidélité et à les inciter à dépenser sont devenus monnaie courante.

Du côté de la demande, les voyages sont de duree plus courte, mais sont plus fréquents. Les voyages d'agrément continuent de représenter la plus grande partie de l'ensemble des voyages, même si les voyages d'affaires ont crû de façon plus rapide. Comme c'est le cas du côté de l'offre, il y a eu des changements importants et appréciables dans le comportement et les préferences des voyageurs.

Étant donné qu'elle repose sur les résultats de la National Travel Survey qui constitue le gros du travail du U.S. Travel Data Center et qui est une enquête téléphonique mensuelle menée auprès d'échantillons probabilistes nationaux de 1500 résidents adultes des Etats contigus des États-Unis, 1'analyse présentée ici portera essentiellement sur la demande des voyages et du tourisme. En plus d'un survol historique des tendances observées au niveau des voyages, nous analyserons le profil des voyageurs américains au plan des voyages d'affaires et des voyages d'agrément.

\footnotetext{
Elizabeth Stewart eat analyate au U.S. Travel Data Center. Ce texté, scrit pour Teorós, a étó traduit do I'anglais avee l'aceord de l'auteure.
}

\section{Caractéristiques générales des voyages effectués par les Améri- cains}

La US Travel and Tourism Administration estime que 1,3 milliard de voyagespersonnes ont été effectués en 1989 , dont environ $96,8 \%$ à l'intérieur des États-Unis et $3,2 \%$ à destination du Canada, du Mexique ou du reste du monde. Le volume des voyages, exprimé en voyages-personnes, augmente depuis 1985 suivant de près le cours extraordinaire de la reprise économique.

De 1980 a 1989, le volume des voyages, exprimé en voyages-personnes, a augmenté de $20 \%$. Cette croissance est toutefois plutôt attribuable à la vigueur de la demande de voyages des cinq dernières années de cette période de dix ans qu'à la vigueur de la demande de voyages enregistrée sur toute la période. Au début des années 1980, la récession a eu uneffet négatif sur le volume des voyages tandis que le nombre de voyages-personnes a baissé de $3 \%$ tous les ans entre 1979 et 1984 . A partir de 1985, une fois que la demande refoulée de biens et services a été satisfaite, la demande de voyages a repris de façon marquée, tandis que les voyages-personnes effectués parles résidents américains ont augmenté à un taux annuel d'à peu près $5 \%$ de 1985 à 1989.

Durant les années 1980 , les voyages d'affaires ont augmenté à un taux annuel de plus de $3 \%$, dépassant la croissance des voyages d'agrement (voir tableau 1). Le gros de la croissance des voyages d'affaires a été enregistré au cours des cinq demières années quand le nombre de voyages d'affaires a crû au taux annuel ahurissant de $8,6 \%$. Ce phénomène est attribuable en grande partie à l'optimisme répandu des milieux d'affaires résultant des conditions économiques favorables. Par exemple, au cours des cinq dernières années de cette décennie, l'inflation a été relativement modeste, augmentant en moyenne d'à peu près $4 \%$ tous les ans comparativement à des hausses de plus de $7 \%$ entre 1979 et 1984 . Pendant ce temps-là, le PNB réel croissait de façon robuste à un taux annuel de $3,4 \%$, comparativement à $1,9 \%$ au cours des cinq premières années des années 1980. La croissance de l'emploi, qui a été de $3 \%$ par année de 1985 à 1989, a aussi contribué à hausser les revenus et les dépenses de consommation au cours des cinq demières années de la décennie.

Par contre, la croissance des voyages d'agrément a été assez modéréc. De 1979 à 1984, les voyages d'agrément ont affiché des baisses annuelles moyennes d'aे peu près $4 \%$. Malgré une reprise au cours des cinq dernières années de la décennie quand leur nombre a augmenté de $5 \%$ par année, les voyages d'agríment ont crû de moins d'un pour cent par année dans l'ensemble sur toute la décennie. Toutefois, comme les voyages d'agrément représentent plus de deux tiers du nombre total de voyages, toute augmentation de son volume, si minime soit-elle, influe beaucoup sur les tendances globales.

Un sous-groupe important des voyages d'agriment, celui des voyages à des fins de vacances, est demeuré stable au cours de la demière décennie, croissant de $2 \%$ par année. Comme les vacances font presque partie intégrante du style de vie nordaméricain, la conjoncture économique du début des années 1980 n'a pas influé sur la croissance des voyages à des fins de vacances aussi négativement que sur la croissance des autres types de voyages. De 1979 a 1984, les voyages a des fins de vacances ont baissé de moins d'un pour cent par année. A partir du milieu de la décennie, la croissance annuelle des voyages à des fins de vacances a été en moyenne de $5 \%$, facilité principalement par l'optimisme renouvele des consommateurs et toute une série d'incitations aux voyages.

La vigueur des voyages à des fins de vacances est toujours évidente l'été, leur saison de pointe. Au cours de cette période, août a été le mois préféré jusqu'en 1987; après quoi juillet est devenu plus populaire. En 1989, les voyages effectués à des fins de vacances d'été $n$ 'ont pas affiché la même croissance robuste qu'au cours des années passées. Toutefois, le volume des voyages àdes fins de vacances demeure relativement élevé et, par conséquent, ils continuent de représenter la base des activités de l'industrie.

Nos données amènent aussi à penserque les consommateurs prennent moins de temps 
de vacances qu'avant. En 1985, la durée moyenne des voyages à des fins de vacances était de 5,7 nuits, mais n'a pas cessé de baisser depuis. En 1989, la durée moyenne des voyages à des fins de vacances avait baissé à 4,7 nuits. Il semble que les consommateurs prennent des vacances plus fréquentes, mais plus courtes.

La propension à prendre des vacances plus courtes est responsable de la popularité des voyages d'une fin de semaine qui ont eu beaucoup de succès au cours de la décennie (voir tableau 2). Les voyages d'une fin de semaine ont été la réponse aux besoins des consommateurs sensibles au temps. Les

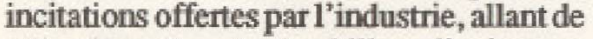
réductions du prix des billets d'avion sur une fin de semaine à des rabais sur le prix des chambres d'hôtel, ont aussi contribue à favoriser ce type de voyages ces dernières années.

Les développements positifs dans les divers sous-groupes du secteur des voyages d'agrément, en particulier au cours de la deuxième moitié de la décennie, peuvent aussi être attribues au fait que l'industrie a réagi efficacement aux demandes très diverses des consommateurs, axant son marketing sur l'acquisition de créneaux. Dans l'ensemble, les consommateurs ont profité de la concurrence auniveau del'offre des biens et services de voyages et de la conjoncture économique favorable. Aussi, les voyages d'agrément tout comme les voyages d'affaires ont prospéré, en particulier au cours des cinq dernières années de la décennie 1980 .

\section{Les voyages d'agrément}

Pour bien évaluer les tendances dans l'industrie des voyages, il est essentiel de faire la distinction entre les divers motifs pour lesquels les voyages sont faits. De cette façon, on peut mesureret comparer les profils des voyageurs et leurs caractéristiques touristiques.

Les voyages à des fins d'agrément (raison principalepour laquelle les gens voyagent), poursuivent une variétéde buts. Les voyages d'agrément englobent les voyages à des fins de vacances, les voyages à des fins de divertissement ou à des fins de loisirs en pleinair, lesexcursionsd'une finde semaine et les autres voyages de ce genre. Par conséquent, même à l'intérieur de ce segment, il y a des différences et des similitudes entre les voyageurs. Toutefois, pour simplifier et pour faciliter la comparaison avecles voyages d'affaires, la presente analyse portera seulement sur l'ensemble des voyages d'agrément.

\section{Caractéristiques}

En 1988,928 millionsdevoyages-personnes ont été effectués à des fins de vacances, ce qui représente une augmentation de $21 \%$ par rapport à 1980 et de $3 \%$ par rapport à 1987. Selon les premières estimations pour 1989 (voir tableau 3), la croissance des voyages d'agrement, quoique modeste, se continuerait. La plupart de ces voyages ont été effectués dans le but de visiter des parents ou des amis, comme l'ont indiqué $46 \%$ des répondants à l'enquête. Les voyages à des fins de divertissement deviennent populaires eux aussi, puisque $34 \%$ des répondants à l'enquête ont déclaré que celaétait le but premier de leur voyage d'agrément. En 1987, seulement $29 \%$ des répondants avaient déclaré avoir effectué des voyages à des fins de divertissement, ce quireprésentait une baisse comparativement à $31 \%$ en 1984.

Comme la majorité des voyageurs d'agrếment ont déclaré avoir été en voyage principalement pour visiter des parents ou des amis, il n'est pas surprenant qu'un grand nombre d'entre eux soient descendus chez leurs parents ou amis. En $1988,44 \%$ des voyageurs d'agrement ont logé chez des amis ou des parents, tandis qu'un pourcentage presque égal ont logé dans des endroits d'hébergement payants, comparativement à la moitié environ et à seulement $40 \%$ respectivement en 1984 . L'accroissement du nombre de voyageurs choisissant de loger dans des endroits d'bébergement payants ces demières années est attribuable aux nombreux rabais offerts par les chaînes d'hôtels à mesure qu'elles fusionnaient et grossissaient.

Les voyages à des fins de vacances sont demeunes stables, tandis qu'un peu plus de $80 \%$ des voyageurs d agrémenten 1988 ont indiqué qu"ils avaient voyagé à des fins de vacances. Ces cinq dernierres années, on peut voir que ce pourcentage est stable tout comme celui des voyages d'une fin de semaine. Les personnes qui partent en voyage d'une fin de semaine représentent à peu près $50 \%$ des voyageurs d'agrément.

Les voyageurs d'agrément se déplacent en général en groupe. A peu prìs $80 \%$ d'entre eux voyagent en groupe de deux personnes ou plus, l'automobile étant le mode de transport préféré. Comme $80 \%$ des voyageursd'agrémentsontplus susceptibles d'utiliserl'automobile, la distance moyenne aller-retour des voyages d'agrément, qui est de 940 milles $(1,560 \mathrm{~km})$, est beaucoup plus petite que celle des voyages effectués parles voyageurs d'affaires. Cette distance moyenne représente toutefois une augmentation par rapport à la distance moyenne de 810 milles $(1,340 \mathrm{~km})$ enregistrée en 1984, tandis que des prix énergétiques plus favorables et la construction d'automobiles économiques en essence et de conception ergonomique favorisent les voyages de longue distance.

Une autre tendance observee récemment dans les voyages d'agrémentest la fréquence des voyages d'agrêment de moins de 24 heures. En 1984, 4\% des voyageurs d'agrément sont partis pour moins d'une journée. En 1987, ce pourcentage avait augmenté à $7 \%$ et il s'établissait à $11 \%$ en 1988. Malgré qu'elle soit encore relativement négligeable en termes relatifs, la hausse régulière au fil des ans s'est effectuée au détriment des voyages d'une nuit ou plus chez des amis ou des parents et des voyages au cours desquels des camionnettes de camping, des roulottes, des véhicules de camping ou des résidences secondaires sont utilisés comme endroits d'hébergement pour la nuit. Les occasions de faire des voyages de moins d'une joumée ont été favorisées par les progrès réalisés dans le secteur des transports comme le développement des réseaux routiers, des services de transport par train et par autobus plus rapides et un plus grand nombre de vols réguliers vers les grands centres de transit aे forte affluence.

Les voyages d'agrément sont plus courts en général et plus fréquents et, parallèlement, leur durce moyenne a baissé. En 1984, la durée moyenne était de 5,6 nuits, comparativement a 4,6 nuits en 1988 (voir tableau 4). Tandis que le marché des voyages de courte durée a crû ces dernières années, celui des voyages de longue durée est demeuré stable à toutes fins pratiques.

En ce qui concerne les endroits de destination, les voyageurs $d^{\prime}$ agrément américains ont montré une forte préférence pour la région Atlantique-Sud (20\%) qui comprend le Delaware, le District de Colombia, la Floride, la Georgie, le Maryland, la Caroline du Nord, la Caroline duSud, la Virginie et la Virginie de l'Ouest. Cette région est suivie de près par la région du Pacifique (15\%) qui comprend 1'État de Washington, 1'Orégon, la Californie, 1'Alaska et Hawaï. En demière position vient la Nouvelle-Angleterre, avec seulement $4 \%$ des voyageurs. Comme une très grande proportion des voyages sont intra-régionaux, cette proportion allant de $50 \%$ à un peu plus de $70 \%$, les activités économiques dans ces régions et la propension des résidents à faire des voyages influe beaucoupsurlevolume des voyageurs dénombrés dans chaque région. 
Profil démographique

Les voyageurs d'agrément américains sont plus susceptibles d'être dans la tranche d'âge des 18 à 54 ans, le gros d'entre eux étant concentrés dans le groupe d'åge des 25 à 44 ans, la génération du "baby boom". Plus de la moitié sont mariés $(58 \%)$ et la très grande majorite $(71 \%)$ sont propriétaires. Seulement $3 \%$ des voyageurs vivent dans des ménages composés d'un adulte seul avec des enfants, tandis que $42 \%$, la plus grande proportion, vivent dans des ménages où il y a au moins deux adultes sans enfant. Parailleurs, $35 \%$ des voyageurs d'agrement. vivent dans des ménages où il $\mathrm{y}$ a au moins deux adultes avec des enfants et seulement $19 \%$ declarent habiter dans des ménages constitués d'un seul adulte seulement.

En ce qui concerne les groupes de professions, les résultats indiquent qu'en 1988 , les cols bleus et les techniciens représentaient une proportion assez importante des voyageurs d'agrément ( $18 \%$ dans les deux cas). Les directeurs et les professionnels representaient $14 \%$ des voyageurs d'agrément, les employés et les vendeurs, $14 \%$, et lespersonnes à la retraite, $15 \%$. Selon les niveaux de revenus cette fois, plus de $60 \%$ des répondants ayant fait des voyages d'agrément avaient un revenu familial annuel d'au moins 10000 (U.S.) mais d'au plus $40000 \$$, tandis que $44 \%$ gagnaient entre $20000 \$$ et $39999 \$$ par année. Seulement $19 \%$ avaient un revenu familial total d'au moins $50000 \$$ parannée.

\section{Les voyages d'affaires}

Les voyages d'affaires effectués par les résidents americains ont affiché une forte hausse au cours des années 1980. Après avoir baissé en 1983 et 1984, ils ont rebondi en 1985 et n'ont pas cessé d'augmenter depuis. Pour 1989, les estimations initiales indiquent que 236 millions de voyagespersonnes auraient été effectués pour des raisons d'affaires, ce qui représente une augmentation de $11 \%$ par rapport à 1988 où 212 millions de voyages-personnes ont été enregistrés.

Les voyages d'affaires englobent les voyages effectués pour se rendre à des réunions, a des congrès ou à des foires commerciales, les visites de représentants, les voyages effectués pour donner des services de consultant, et les voyages effectués pour diverses autres raisons connexes. Leur frêquence est souvent liée à la rentabilité de lasociété etpar conséquent, indirectement au climat global des affaires. Aussi, les voyages d'affaires peuvent facilement être reportés ou annulés lorsque
TABLEAU 1

Les voyages d'affaires augmentent deux fois plus vite que les voyages d'agrément
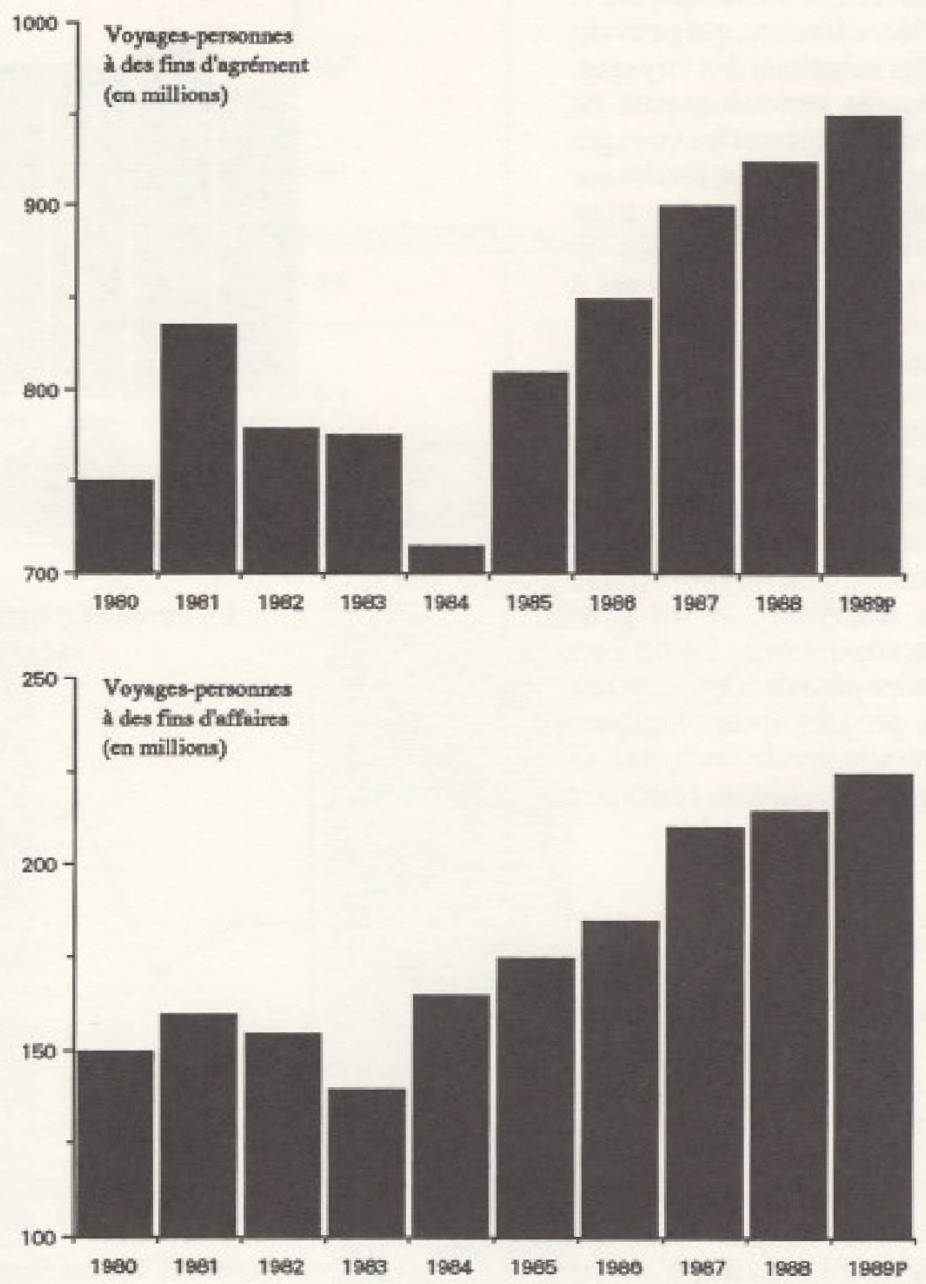

$1949=$ pnojection

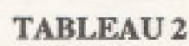

Les voyages à des fins de vacances d'une fin de semaine entre 1980 et 1989

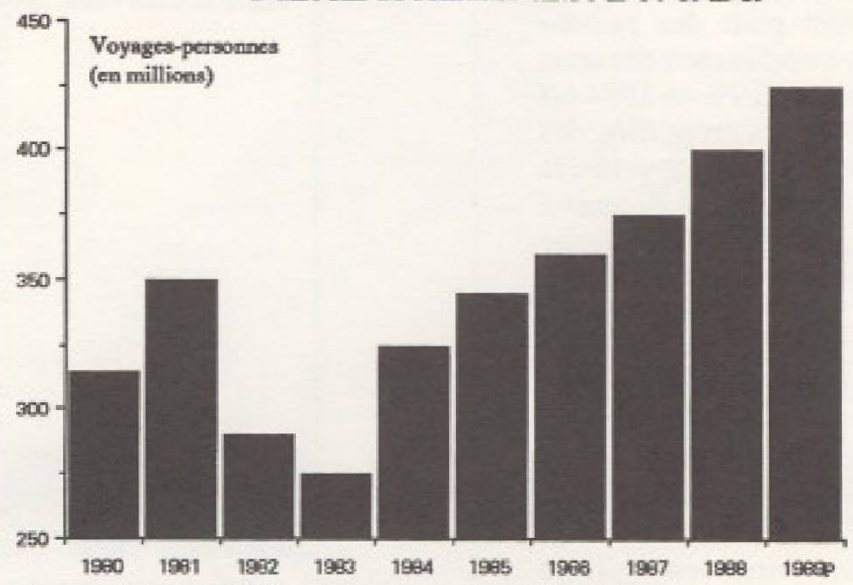

Source: National Travel Survey du US Travel Data Center. 
la conjoncture économique est défavorable, surtout depuis qu'on est dans l'ère des communications électroniques.

Fait surprenant, leur extraordinaire croissance a surtout eu lieu depuis l'avènement des services de livraison du courrier le jour suivant, des télécopieurs et des services de téléconférence, qui peuvent être vus comme des substituts des voyages. En effet, ces progrès technologiques ne peuvent pas toujours remplacer les voyages d'affaires qu'il faut faire pour se rendre sur place en personne, par exemple, pour participer à des foires commerciales ou assister à des congrès, qui commencent à constituer une part importante des voyages d'affaires, représentant à eux seuls plus des deux tiers des voyages d'affaires effectués par les voyageurs d'affaires au cours de n'importequellepériode de 12 mois donnée. Demême, les réunions du personnel vendeur et les visites de représentants ne peuvent pasêtre aussi efficaces, comme une réclame publicitaire à la télévision, si les gens n'apparaissent pasenpersonne. Loind'être dessubstituts dans les périodes de prospérité économique, les progrès technologiques semblent au contraire avoir complété et facilité les voyages d'affaires au cours des années 1980.

\section{Caractéristiques}

Selon les données de 1988, les données détaillées les plus récentes sur les caractéristiques des voyages, la majorité des 212 millions de voyages-personnes effectués pour des raisons d'affaires ou pour assister à des congrès comportaient des séjours d'une nuit ou plus dans des endroits d'hébergement payants, à savoir des hôtels ou des motels $(67 \%)$. Toutefois, comme dans le cas des voyages d'agrément, les voyages de moins d'une journée sont de plus en plus populaires comparativement aux voyages comportant des séjours chez des amis ou des parents. La proportion des Americains voyageant pour des raisons d'affaires qui sont descendus chez des amis ou des parents qui était de $19 \%$ en 1984 est tombee à $14 \%$ en 1988. La proportion des répondants qui ont déclaré être revenus la journée même sans avoir passé de nuit à l'extérieurpendant qu'ils étaient en voyage d'affaires a augmenté de $8 \%$ en 1984 à $19 \%$ en 1988.

Comme on l'a dit précédemment, les voyages d'affaires peuvent englober des activités très diverses. Selon les résultats de la 1988 Survey of Business Travelers menée par le US Travel Data Center et parrainée par Reed International, la participation à des réunions, congrès ou foires commerciales a souvent été citée
TABLEAU 3

Les voyages d'agrément ont augmenté d'à peu près $25 \%$ au cours des années 1980

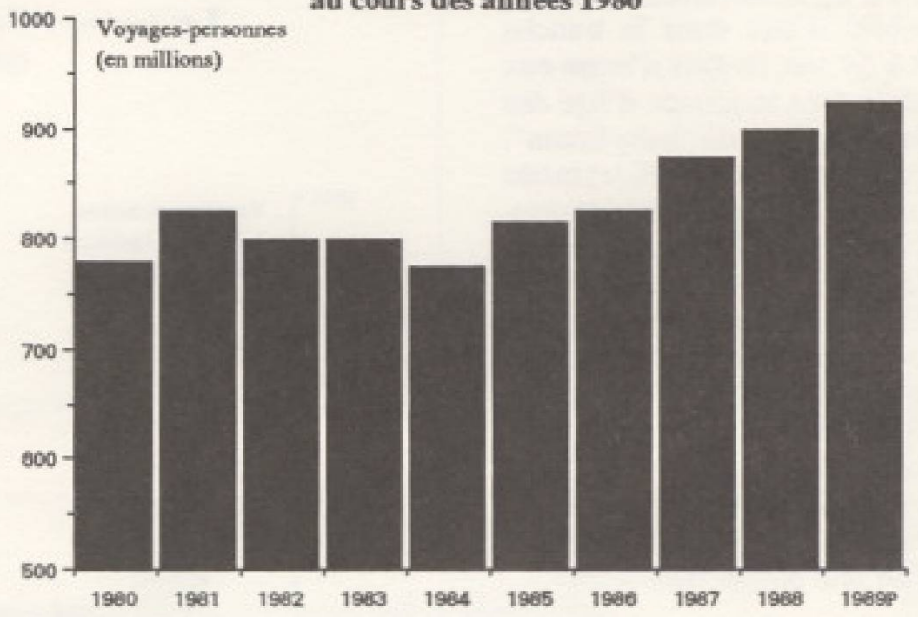

Source: US Travel Data Cenier.

$1089=$ projection

\section{TABLEAU 4}

La durée moyenne des voyages-nuitées à des fins de vacances continue de balsser

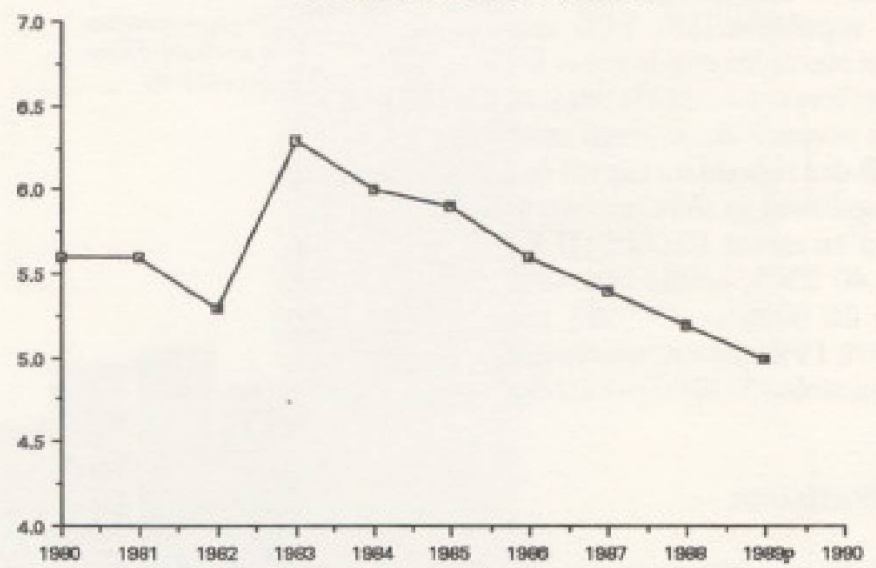

Source: National Travel Survey du US Travel Data Center.

$19 a 9$ a projuction

\section{TABLEAU 5}

Tous les types de voyages ont augmenté dans les années 1980
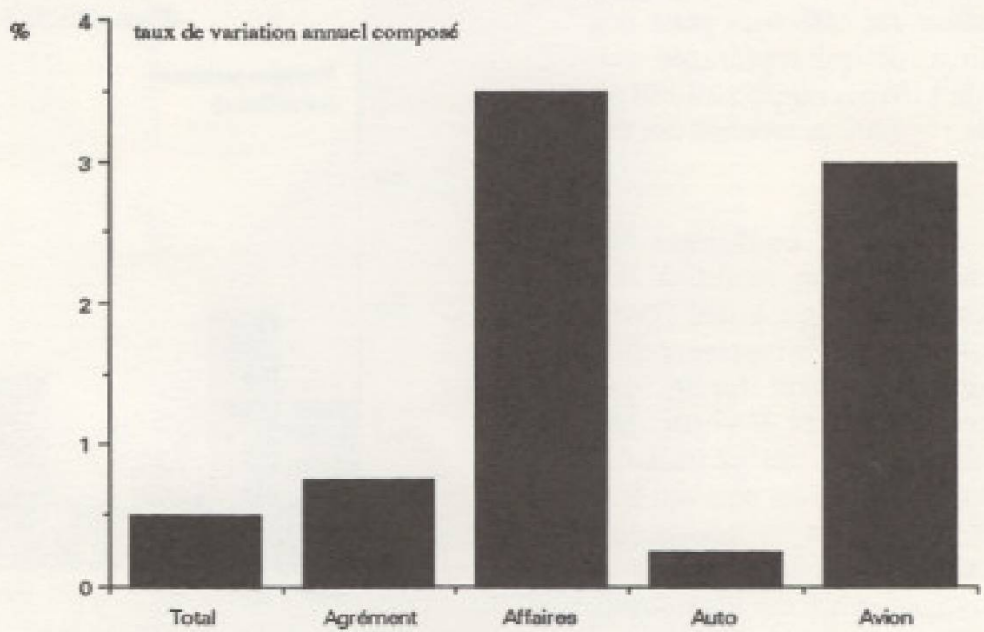

Source: US Travel Data Center. 
comme la raison principale d'un voyage d'affaires $(43 \%)$. Les visites de représentants suivent de près tout comme les voyages pour des raisons d'affaires personnelles ( $12 \%$ dans les deux cas).

En ce qui conceme le mode de transport, les voyages d'affaires par automobile sont encore plus fréquents que les voyages par avion ou par d'autres modes de transport. En $1988,57 \%$ des voyages-personnes à des fins d'affaires comportaient l'utilisation d'une voiture, d'un camion ou d'unvéhicule de plaisance et $41 \%$ ont été faits par avion. La proportion des voyages d'affaires effectués par automobile a été relativement stable ces dernières années. Les voyages d'affaires effectués par avion, par contre, ont augmenté de $37 \%$ en 1984 à $41 \%$ en 1988 , avec le résultat que la distance moyenne desvoyages d'affaires a augmenté de $950 \mathrm{a} 1100$ milles $(1,570 \mathrm{~km}$ \1,820 km) au cours de cette période.

Du point de vue de la durée, les voyages d'affaires ont suivi les mêmes tendances que les voyages d'agrément. La durée moyenne des voyages a baissé de 4,5 nuits en 1984 à 3,3 nuits en 1988. Ce phénomène reflète en partie les horaires plus charges des compagnies d'aviation qui leur permettent d'offrir des services plus fréquents entre les destinations et la popularité croissante des voyages de courte durée, notamment des voyages de moins d'une joumée.

\section{Profil démographique}

Les voyageurs d'affaires américains sont surtout des hommes $(71 \%)$, des personnes maries ( $62 \%)$ et se retrouvent surtout dans les catégories de professions liberrales et de gestion et de professions techniques (30\% et $31 \%$ respectivement). Une majorité de voyageurs d'affaires américains avaient un revenu familial annuel situé entre $50000 \$$ (U.S.) et $74999 \$$ en $1988(21 \%)$, tandis que $11 \%$ gagnaient au moins $100000 \$$. Environ $40 \%$ résidaient dans des ménages où deux des membres gagnent un salaire.

La structure de ménage des voyageurs d'affaires ressemble beaucoup à celle des voyageurs d'agrément. Les ménages avec des enfants et avec, au moins, deux adultes dominent dans la catégorie des voyages d'affaires (38\%), suivis des ménages avec deux ou plus de deux adultes seulement (37\%).

Les voyages d'affaires sont très concentrés chez les 55 ans et moins. Les voyageurs d'affaires dans leurs meilleures années de travail, c'est-à-dire entre 35 et 54 ans, représentent à peu près $50 \%$ des voyages- personnes effectués pour des raisons d'affaires, tandis qu'environ $11 \%$ sont dans le groupe d'âge des 55 à 74 ans.

\section{Conclusion}

Les voyages d'affaires et les voyages d'agrement ont profite des incitations offertes par l'industrie des voyages et des conditions économiques généralement bonnes qui influent sur la confiance des consommateurs et des gens d'affaires. Le but du voyage, toutefois, détermine les préférences des consommateurs quand ils décident de voyager. Par exemple, les voyageurs d'affaires sont plus susceptibles de séjourner à l'hôtel ou dans un motel, tandis que les voyageurs d'agrément sont plus susceptibles de loger chez des amis ou des parents. Comme les frais d'hébergement à l'hôtel ou dans un motel entrent dans les dépenses d'entreprise et sont donc payés par la société, il est normal que près de $70 \%$ des voyages-personnes effectués pour des raisonsd'affaires aient comportédes séjours d'une nuit ou plus dans des endroits d'hébergement payants. Par contre, les voyages d'agrément sont généralement des dépenses personnelles, à moins d'être combinés avec un voyage d'affaires, et les voyageurs d'agrement sont donc moins incités à utiliser des endroits d'hébergement payants. Mais les rabais consentis par les hôtels pourraient contrebalancer ce phenomène et les tendances observées jusqu'ici montrent que, malgré qu'elle soit moins populaire que le séjour chez desamis ou des parents, l'utilisation de l'hôtel chez les voyageurs d'agrément est en hausse.

Du point de vue de la durée des voyages, les voyageurs d'affaires partenten général pour des périodes moins longues que les voyageurs d'agrément. En 1988, la durée des voyages d'affaires s'est établie en moyenne à 3,3 nuits, comparativement a 4,6 nuits pourles voyages d'agrément. Cette différence reflète en partie le fait que les voyageursd'affaires ont plus de contraintes de temps que les voyageurs d'agrément.

Comme les voyageurs d'affaires sont plus susceptibles de voyager par avion que les voyageurs d'agrément ( $41 \%$ contre $16 \%$ ), ladistance qu' ils parcourent est plus longue aussi. En 1988, les voyages d'affaires s'établissaient en moyenne à 1100 milles $(1,820 \mathrm{~km})$, comparativement à 940 milles $(1,560 \mathrm{~km})$ pour les voyages d'agrément où le transport par automobile dominait. Dans les deux cas, la distance moyenne a augmenté au fil des ans, probablement à cause des rabais consentis sur les tarifs aériens, du plus grand nombre de vols réguliers et des automobiles plus confortables et plus économiques, comme on l'a déjà dit.

En ce qui concerne l'aspect démographique, un plus grand pourcentage d'hommes que de femmes voyagent par affaires, mais cette différence n'est pas aussi évidente dans les voyages d'agrément. À mesure que les femmes entrent dans la population active et commencent a occuper des postes qui obligent à voyager, il est vraisemblable qu'elles deviendront une forcemajeure dans les voyages d'affaires. Dans le 1988 Survey of Business Travelers, les données ont montré que $26 \%$ des femmes qui voyagent par affaires sont des professionnelles ou des gestionnaires, comparativement aे $31 \%$ des hommes qui voyagent par affaires, même si, comme voyageurs, les femmes représentent un plus gros pourcentage que les hommes dans certaines industries comme les finances, les banques, les assurances, la santé, les services juridiques, entre autres.

Même si le profil des voyageurs d'affaires et celui des voyageurs d'agrément peuvent différer à certains égards, ils peuvent se ressembler à d'autres égards. Les deux types de voyageurs sont très concentrés dans le groupe d'àge des 25 a 44 ans ( $60 \%$ des voyageurs d'affaires et $46 \%$ des voyageurs d'agrément), ils sont plus susceptibles d'être mariés ( $62 \%$ et $58 \%$ respectivement) et plus susceptibles d'être propriétaires $(74 \%$ et $70 \%$ respectivement).

Pour résumer, les voyages d'affaires et les voyages d'agrément sont deux segments distincts de l'industrie des voyages. Pourtant, la croissance dans l'un et l'autre segment contribue de façon appréciable aux tendances observées de façon globale dans les voyages. Mais plus important encore, dans les deux cas, la croissance au fil des ans est grandement influencee par les mouvements démographiques ainsi que par $1^{\prime}$ évolution de la conjoncture économique et de l'offre sur le marché des voyages. $f$ 